\title{
MAGNETO-MICROPOLAR FLUID MOTION: GLOBAL EXISTENCE OF STRONG SOLUTIONS
}

\author{
ELVA E. ORTEGA-TORRES AND MARKO A. ROJAS-MEDAR
}

Received 1 December 1997

By using the spectral Galerkin method, we prove a result on global existence in time of strong solutions for the motion of magneto-micropolar fluid without assuming that the external forces decay with time. We also derive uniform in time estimates of the solution that are useful for obtaining error bounds for the approximate solutions.

\section{Introduction}

In this work, we will be concerned with global existence in time of strong solutions to the three dimensional magneto-micropolar fluid equations. Being $\Omega \subset \mathbb{R}^{3}$ a $C^{1,1}$ regular bounded open set, $T>0$ these equations are (see [1]):

$$
\begin{gathered}
\frac{\partial u}{\partial t}+u \cdot \nabla u-(\mu+\chi) \Delta u+\nabla\left(p+\frac{1}{2} r b \cdot b\right)=\chi \operatorname{rot} w+r b \cdot \nabla b+f, \\
j \frac{\partial w}{\partial t}+j u \cdot \nabla w-\gamma \Delta w+2 \chi w-(\alpha+\beta) \nabla \operatorname{div} w=\chi \operatorname{rot} u+g, \\
\frac{\partial b}{\partial t}-v \Delta b+u \cdot \nabla b-b \cdot \nabla u=0, \\
\operatorname{div} u=\operatorname{div} b=0 \quad \text { in }(0, T) \times \Omega .
\end{gathered}
$$

Here, $u(t, x) \in \mathbb{R}^{3}$ denotes the velocity of the fluid at a point $x \in \Omega$ and time $t \in[0, T] ; w(t, x) \in \mathbb{R}^{3}, b(t, x) \in \mathbb{R}^{3}$ and $p(t, x) \in \mathbb{R}$ denote, respectively, the micro-rotational velocity, the magnetic field and the hydrostatic pressure; the constants $\mu, \chi, \alpha, \beta, \gamma, j$, and $v$ are positive numbers associated to properties of the material; $f(t, x), g(t, x) \in \mathbb{R}^{3}$ are given external fields.

We assume that on the boundary $\partial \Omega$ of $\Omega$ the following conditions hold

$$
u(t, x)=w(t, x)=b(t, x)=0, \quad(t, x) \in[0, T] \times \partial \Omega,
$$

Copyright $\odot 1999$ Hindawi Publishing Corporation

Abstract and Applied Analysis 4:2 (1999) 109-125

1991 Mathematics Subject Classification: 35Q35, 76M30, 76W05

URL: http://aaa.hindawi.com/volume-4/S1085337599000287.html 
we have assumed homogeneous boundary conditions just for simplicity. In standard ways the nonhomogeneous case could be treated. The initial conditions are

$$
u(0, x)=u_{0}(x), \quad w(0, x)=w_{0}(x), \quad b(0, x)=b_{0}(x), \quad x \in \Omega .
$$

Equations (1.1a) has the familiar form of the Navier-Stokes equations but it is coupled with (1.1b), which essentially describes the motion inside the macrovolume as they undergo micro-rotational effects represented by the micro-rotational velocity vector $w$. For fluids with no micro-structure this parameter vanishes. For Newtonian fluids, equation (1.1a) and (1.1b) decouple since $\chi=0$.

When the magnetic field is absent $(b \equiv 0)$, problem (1.1), (1.2), and (1.3) was studied by Lukaszewicz [6, 7], Galdi and Rionero [4], and Padula and Russo [8]. Lukaszewicz [6] established the global existence of weak solutions for (1.1), (1.2), and (1.3) under certain assumptions by using linearization and an almost fixed point theorem. By using the same technique, Lukaszewicz [7] proved the local and global existence, and also the uniqueness of strong solution. Galdi and Rionero [4] established results similar to the Lukaszewicz [7]. Padula and Russo [8] studied the uniqueness of the solutions for problem (1.1), (1.2), and (1.3) in unbounded domains.

The full system (1.1), (1.2), and (1.3) was studied by Galdi and Rionero [4], and they stated, without proof, results of existence and uniqueness of strong solutions. Ahmadi and Shaninpoor [1] studied the stability of solutions for the system (1.1), (1.2), and (1.3). The more constructive spectral Galerkin method was used by Rojas-Medar [9] to obtain local in time strong solutions.

In this paper, we will consider the global existence of strong solutions of (1.1), (1.2), and (1.3), with homogeneous boundary condition for $u, w$, and $b$ for simplicity of exposition. Thus, the results in this paper form the theoretical basis for future numerical analysis of the problem: here we will obtain estimates for the approximate solutions that will be fundamental in a forthcoming paper in which we will obtain uniform error estimates for such Galerkin approximations.

We observe that all known results on global existence of strong solutions for the system (1.1), (1.2), and (1.3) require some short of decay in time of the associated external forces.

However, in the case of the classical Navier-Stokes equations $(w \equiv 0, b \equiv 0)$, this kind of decay requirement is not necessary (see, for instance, Heywood and Rannacher, [5]). Therefore, one should be able to prove the global existence without this decay condition in the case of equations (1.1), (1.2), and (1.3).

This is indeed true, and we shall prove it under certain regularity assumptions on the initial data and external forces. This proof will be the main result of the present article. In particular, we will require smallness of the $H^{1}$-norm of the initial data, as well as, of the $L^{\infty}\left([0, \infty) ; L^{2}(\Omega)\right)$-norm of the forces.

Thus, we research basically the same level of knowledge as the one in the case of the classical Navier-Stokes equations.

Finally, the paper is organized as follows: in Section 2, we state the basic assumptions and results that will be used later in the paper; we also rewrite (1.1), (1.2), and (1.3) in a more suitable weak form; we describe the approximation method and state the results 
of local existence (Theorems 2.3, 2.4, and 2.5). In Section 3, we give and prove the global existence theorem analogous to Theorem 2.3 (Theorem 3.1). In Section 4, we give and prove the regularity result (Theorem 4.1). In Section 5, we give the results on pressure, and finally in Section 6, we give results of global existence and regularity when assuming exponential decay in time of the associated external forces.

\section{Statements and notations}

Let $\Omega \subset \mathbb{R}^{3}$, be a bounded domain with boundary $\partial \Omega$ of class $C^{1,1}$. Let $H^{s}(\Omega)$ be the usual Sobolev spaces on $\Omega$ with norm $\|\cdot\|_{H^{s}}(s$ real $) ;(\cdot, \cdot)$ denotes the usual inner product in $L^{2}(\Omega)$ and $\|\cdot\|$ the $L^{2}$-norm on $\Omega$. By $H_{0}^{1}(\Omega)$ we denote the completion of $C_{0}^{\infty}(\Omega)$ under the norm $\|\cdot\|_{H^{1}}$; the $L^{p}$-norm on $\Omega$ is denoted by $\|\cdot\|_{L^{p}}, 1 \leq p \leq \infty$. If $B$ is a Banach space, we denote by $L^{q}(0, T ; B)$ the Banach space of the $B$-valued functions defined in the interval $(0, T)$ that are $L^{q}$-integrable in the sense of Bochner.

The functions, in this paper, are either $\mathbb{R}$ or $\mathbb{R}^{n}$-valued and we will not distinguish them in our notations; the situation will be clear from the context.

We shall consider the following spaces of divergence-free functions (see Temam [10])

$$
\begin{gathered}
C_{0, \sigma}^{\infty}(\Omega)=\left\{v \in C_{0}^{\infty}(\Omega) ; \operatorname{div} v=0 \text { in } \Omega\right\}, \\
H=\text { closure of } C_{0, \sigma}^{\infty}(\Omega) \text { in } L^{2}(\Omega), \\
V=\text { closure of } C_{0, \sigma}^{\infty}(\Omega) \text { in } H^{1}(\Omega) .
\end{gathered}
$$

We observe the space $V$ is characterized by

$$
V=\left\{u \in H_{0}^{1}(\Omega) ; \operatorname{div} u=0 \text { in } \Omega\right\} .
$$

The space $L^{2}(\Omega)$ has the decomposition $L^{2}(\Omega)=H \oplus H^{\perp}$, where $H^{\perp}=\{\phi \in$ $L^{2}(\Omega) ; \exists p \in H^{1}(\Omega)$ with $\phi=\nabla p$ \} (Helmholtz decomposition).

Throughout the paper, $P$ will denote the orthogonal projection from $L^{2}(\Omega)$ onto $H$. Then the operator $A: D(A) \hookrightarrow H \rightarrow H$ given by $A=-P \Delta$ with domain $D(A)=$ $H^{2}(\Omega) \cap V$ is called the Stokes operator. It is well known that the operator $A$ is positive definite, self-adjoint operator and is characterized by the relation

$$
(A w, v)=(\nabla w, \nabla v) \quad \forall w \in D(A), v \in V .
$$

The operator $A^{-1}$ is linear continuous from $H$ into $D(A)$, and since the injection of $D(A)$ in $H$ is compact, $A^{-1}$ is a compact operator in $H$. As an operator in $H, A^{-1}$ is also self-adjoint. By a well-known theorem of Hilbert spaces, there exists a sequence of positive numbers $\mu_{j}>0, \mu_{j+1} \leq \mu_{j}$ and an orthonormal basis of $H,\left\{\varphi^{j}(x)\right\}_{j=1}^{\infty}$ such that $A^{-1} \varphi^{j}=\mu_{j} \varphi^{j}$. We denote by $\lambda_{j}=\mu_{j}^{-1}$. Since $A^{-1}$ has range in $D(A)$ we obtain that

$$
A \varphi^{j}=\lambda_{j} \varphi^{j}, \quad \varphi^{j} \in D(A)
$$


112 Magneto-micropolar fluid motion: global existence of strong solutions

$0<\lambda_{1}<\cdots \leq \lambda_{j} \leq \lambda_{j+1} \leq \cdots, \lim _{j \rightarrow \infty} \lambda_{j}=+\infty$ and $\left\{\varphi^{j}(x)\right\}_{j=1}^{\infty}$ are orthogonal basis of $H$. Therefore, $\left\{\varphi^{j}(x) / \sqrt{\lambda_{j}}\right\}_{j=1}^{\infty}$ and $\left\{\varphi^{j}(x) / \lambda_{j}\right\}_{j=1}^{\infty}$ form an orthogonal basis in $V$ (with the inner product $(\nabla u, \nabla v), u, v \in V)$ and $H^{2}(\Omega) \cap V$ (with inner product $(A u, A v), u, v \in D(A))$, respectively. We denote by $V_{k}=\operatorname{span}\left\{\varphi^{1}(x), \ldots, \varphi^{k}(x)\right\}$.

We observe that for the regularity of the Stokes operator, it is usually assumed that $\Omega$ is of class $C^{3}$, this being in order to use Cattabriga's results [3]. We use, instead, the stronger results of Amrouche and Girault [2] which implies, in particular, that when $A u \in L^{2}(\Omega)$ then $u \in H^{2}(\Omega)$ and $\|u\|_{H^{2}}$ and $\|A u\|$ are equivalent norms when $\Omega$ is of class $C^{1,1}$.

Similar considerations are true for the Laplacian operator $B \equiv-\Delta: D(B) \rightarrow L^{2}(\Omega)$ with the Dirichlet boundary conditions with domain $D(B) \equiv H^{2}(\Omega) \cap H_{0}^{1}(\Omega)$ and we will denote $\phi^{i}(x), \gamma_{i}$ the eigenfunctions and eigenvalues of $B$. We denote by $H_{k}=\operatorname{span}\left\{\phi^{1}(x), \ldots, \phi^{k}(x)\right\}$.

By using the properties of $P$, we can reformulate the problem (1.1), (1.2), and (1.3) as follows: find $u, w, b$, in suitable spaces, to be exactly defined later on, satisfying

$$
\begin{array}{r}
\left(u_{t}, \varphi\right)+(u \cdot \nabla u, \varphi)+(\mu+\chi)(A u, \varphi)=\chi(\operatorname{rot} w, \varphi)+r(b \cdot \nabla b, \varphi)+(f, \varphi), \\
j\left(w_{t}, \phi\right)+j(u \cdot \nabla w, \phi)+\gamma(B w, \phi)+2 \chi(w, \phi)+(\alpha+\beta)(\operatorname{div} w, \operatorname{div} \phi) \\
=\chi(\operatorname{rot} u, \phi)+(g, \phi),
\end{array}
$$

$$
\begin{gathered}
\left(b_{t}, \psi\right)+v(A b, \psi)+(u \cdot \nabla b, \psi)-(b \cdot \nabla u, \psi)=0, \\
\text { for } 0<t<T, \forall \varphi, \psi \in V, \phi \in H_{0}^{1}(\Omega), \\
u(0)=u_{0}, \quad w(0)=w_{0}, \quad b(0)=b_{0} .
\end{gathered}
$$

Now, we define strong solutions of the problem (2.5).

Definition 2.1. Let $u_{0}, b_{0} \in V, w_{0} \in H_{0}^{1}(\Omega)$, and $f, g \in L^{\infty}\left([0, \infty) ; L^{2}(\Omega)\right)$. By a strong solution of the problem (2.5), we mean a triple of vector-valued functions $(u, w, b)$ such that $u, b \in L^{\infty}([0, \infty) ; V) \cap L_{\mathrm{Loc}}^{2}([0, \infty) ; D(A)), w \in L^{\infty}([0, \infty)$; $\left.H_{0}^{1}(\Omega)\right) \cap L_{\text {Loc }}^{2}([0, \infty) ; D(B))$ and that satisfies $(2.5)$.

Remark 2.2. In what follows, we will prove that if $(u, w, b)$ is a strong solution of (2.5), then $u_{t}, b_{t} \in L_{\mathrm{Loc}}^{2}([0, \infty) ; H)$ and $w_{t} \in L_{\mathrm{Loc}}^{2}\left([0, \infty) ; L^{2}(\Omega)\right)$. This condition, together with $u, b \in L_{\text {Loc }}^{2}([0, \infty) ; D(A))$ and $w \in L_{\text {Loc }}^{2}([0, \infty) ; D(B))$, implies by interpolation (see Temam [10, page 260]), that $u, b$ are almost everywhere equal to continuous functions from $[0, T]$ into $V(0<T<\infty)$; analogously $w$ is almost everywhere equal to a continuous function from $[0, T]$ into $H_{0}^{1}(\Omega)(0<T<\infty)$. Consequently, the initial conditions $u(0)=u_{0}, b(0)=b_{0}, w(0)=w_{0}$ are meaningful.

To prove existence of strong solutions we will apply the spectral Galerkin method to (2.5). That is, we consider the finite dimensional subspaces $V_{k}=\operatorname{span}\left[\varphi^{1}, \ldots, \varphi^{k}\right]$, 
$H_{k}=\operatorname{span}\left[\phi^{1}, \ldots, \phi^{k}\right], k \in \mathbb{N}$, the corresponding orthogonal projections $P_{k}: L^{2} \rightarrow V_{k}$ and $R_{k}: L^{2} \rightarrow H_{k}$ and the approximate solutions

$$
\begin{aligned}
& u^{k}(t, x)=\sum_{i=1}^{k} c_{i k}(t) \varphi^{i}(x), \\
& w^{k}(t, x)=\sum_{i=1}^{k} d_{i k}(t) \phi^{i}(x), \\
& b^{k}(t, x)=\sum_{i=1}^{k} e_{i k}(t) \varphi^{i}(x),
\end{aligned}
$$

developed in terms of eigenfunctions of the Stokes and Laplace operators. Then, the coefficients $c_{i k}(t), d_{i k}(t)$, and $e_{i k}(t)$ are found by requiring that $u^{k}, w^{k}$, and $b^{k}$ satisfy the following equations:

$$
\begin{gathered}
u_{t}^{k}+(\mu+\chi) A u^{k}+P_{k}\left(u^{k} \cdot \nabla u^{k}\right)=\chi P_{k} \operatorname{rot} w^{k}+r P_{k}\left(b^{k} \cdot \nabla b^{k}\right)+P_{k} f \\
j w_{t}^{k}+j R_{k}\left(u^{k} \cdot \nabla w^{k}\right)+\gamma B w^{k}+2 \chi w^{k}-(\alpha+\beta) R_{k} \nabla \operatorname{div} w^{k} \\
=\chi R_{k} \operatorname{rot} u^{k}+R_{k} g \\
b_{t}^{k}+v A b^{k}+P_{k}\left(u^{k} \cdot \nabla b^{k}\right)-P_{k}\left(b^{k} \cdot \nabla u^{k}\right)=0 \\
u^{k}(0)=P_{k} u_{0}, \quad w^{k}(0)=R_{k} w_{0}, \quad b^{k}(0)=P_{k} b_{0} .
\end{gathered}
$$

This is equivalent to the weak form

$$
\begin{array}{r}
\left(u_{t}^{k}, \varphi\right)+(\mu+\chi)\left(\nabla u^{k}, \nabla \varphi\right)+\left(u^{k} \cdot \nabla u^{k}, \varphi\right)=\chi\left(\operatorname{rot} w^{k}, \varphi\right)+r\left(b^{k} \cdot \nabla b^{k}, \varphi\right)+(f, \varphi), \\
j\left(w_{t}^{k}, \phi\right)+j\left(u^{k} \cdot \nabla w^{k}, \phi\right)+\gamma\left(\nabla w^{k}, \nabla \phi\right)+2 \chi\left(w^{k}, \phi\right)+(\alpha+\beta)\left(\operatorname{div} w^{k}, \operatorname{div} \phi\right) \\
=\chi\left(\operatorname{rot} u^{k}, \phi\right)+(g, \phi), \\
\left(b_{t}^{k}, \psi\right)+v\left(\nabla b^{k}, \nabla \psi\right)+\left(u^{k} \cdot \nabla b^{k}, \psi\right)-\left(b^{k} \cdot \nabla u^{k}, \psi\right)=0, \\
u^{k}(0)=P_{k} u_{0}, \quad w^{k}(0)=R_{k} w_{0}, \quad b^{k}(0)=P_{k} b_{0}, \quad \forall \varphi, \psi \in V_{k}, \phi \in H_{k} .
\end{array}
$$

By using these approximations, Rojas-Medar [9] proved a local in time existence theorem for (2.5). Their results are the following.

THeOREM 2.3. Let the initial values $u_{0}, b_{0} \in V, w_{0} \in H_{0}^{1}(\Omega)$ and the external forces $f, g \in L^{2}\left(0, T ; L^{2}(\Omega)\right)$. Then, on a (possibly small) time interval $\left[0, T_{1}\right], 0<T_{1} \leq T$, problem (2.5) has a unique strong solution $(u, w, b)$. This solution belongs to $C\left(\left[0, T_{1}\right] ; V\right) \times C\left(\left[0, T_{1}\right] ; H_{0}^{1}(\Omega)\right) \times C\left(\left[0, T_{1}\right] ; V\right)$. 
114 Magneto-micropolar fluid motion: global existence of strong solutions

Moreover, there exists $C^{1}$-functions $F(t), F_{1}(t)$, and $G(t)$ such that for any $t \in$ $\left[0, T_{1}\right]$, there hold

$$
\begin{aligned}
\|u(t)\|^{2} & +j\|w(t)\|^{2}+r\|b(t)\|^{2} \\
& +\int_{0}^{t}\left((\mu+\chi)\|\nabla u(s)\|^{2}+\gamma\|\nabla w(s)\|^{2}+2 r v\|\nabla b(s)\|^{2}\right) d s \\
& +\int_{0}^{t}\left(4 \chi\|w(s)\|^{2}+2(\alpha+\beta)\|\operatorname{div} w(s)\|^{2}\right) d s \leq F(t), \\
\|\nabla u(t)\|^{2} & +\|\nabla b(t)\|^{2}+j\|\nabla w(t)\|^{2} \\
& +c \int_{0}^{t}\left(\|A u(s)\|^{2}+\|A b(s)\|^{2}+\|B w(s)\|^{2}\right) d s \leq F_{1}(t), \\
& \int_{0}^{t}\left(\left\|u_{t}(s)\right\|^{2}+\left\|b_{t}(s)\right\|^{2}+j\left\|w_{t}(s)\right\|^{2}\right) d s \leq G(t) .
\end{aligned}
$$

Also, the same kind of estimates hold uniformly in $k \in \mathbb{N}$ for the Galerkin approximations $\left(u^{k}, w^{k}, b^{k}\right)$.

With stronger assumptions on the initial values and external fields, we are able to prove the following.

THEOREM 2.4. In addition to the assumptions of Theorem 2.3, assume that $u_{0}, b_{0} \in$ $V \cap H^{2}(\Omega), w_{0} \in H_{0}^{1}(\Omega) \cap H^{2}(\Omega)$, and $f_{t}, g_{t} \in L^{2}\left(0, T ; L^{2}(\Omega)\right)$. Then, the solution $(u, w, b)$ satisfies

$$
\begin{aligned}
& \left\|u_{t}(t)\right\|^{2}+j\left\|w_{t}(t)\right\|^{2}+r\left\|b_{t}(t)\right\|^{2} \\
& \quad+c \int_{0}^{t}\left(\left\|\nabla u_{t}(s)\right\|^{2}+\left\|\nabla w_{t}(s)\right\|^{2}+\left\|\nabla b_{t}(s)\right\|^{2}\right) d s \leq H_{0}(t), \\
& \|A u(t)\|^{2}+\|B w(t)\|^{2}+\|A b(t)\|^{2} \leq H_{1}(t), \\
& \int_{0}^{t}\left(\left\|u_{t t}(s)\right\|_{V^{*}}^{2}+\left\|w_{t t}(s)\right\|_{H^{-1}}^{2}+\left\|b_{t t}(s)\right\|_{V^{*}}^{2}\right) d s \leq H_{2}(t),
\end{aligned}
$$

for every $t \in\left[0, T_{1}\right]$, where $H_{i}(t), i=0,1,2$ are continuous functions in $t \in\left[0, T_{1}\right]$. Also

$$
\begin{gathered}
u, b \in C^{1}\left(\left[0, T_{1}\right] ; H\right) \cap C\left(\left[0, T_{1}\right] ; D(A)\right), \\
w \in C^{1}\left(\left[0, T_{1}\right] ; L^{2}(\Omega)\right) \cap C\left(\left[0, T_{1}\right] ; D(B)\right) .
\end{gathered}
$$

Moreover, the same kind of estimates hold uniformly in $k$ for the Galerkin approximations $\left(u^{k}, w^{k}, b^{k}\right)$.

As a consequence of Theorems 2.3 and 2.4, by using the results of Amrouche and Girault [2], we conclude the following theorem. 
THEOREM 2.5. Under the hypotheses of Theorem 2.3, there exist unique function $p \in$ $L^{2}\left(0, T_{1} ; H^{1}(\Omega) / \mathbb{R}\right)$ such that $(u, w, b, p)$ is solution of $(1.1),(1.2)$, and (1.3). Under the hypothesis of Theorem $2.4, p \in L^{\infty}\left(0, T_{1} ; H^{1}(\Omega) / \mathbb{R}\right) \cap C\left(\left[0, T_{1}\right] ; L^{2}(\Omega) / \mathbb{R}\right)$.

Finally, we would like to say that as it is usual in this context, we will denote by $c, C$ generic constants depending at most on $\Omega$ and the fixed parameters in the problem $(\mu, \chi, r, j, v, \alpha, \beta, \gamma)$ and the initial conditions. When for any reason we want to emphasize the dependence of a certain constant on a given parameter we will denote this constant with a subscript.

\section{Global existence}

The analogue to Theorem 2.3 is the following result.

THEOREM 3.1. Let the initial values $u_{0}, b_{0} \in V, w_{0} \in H_{0}^{1}(\Omega)$ and the external forces $f, g \in L^{\infty}\left([0, \infty) ; L^{2}(\Omega)\right)$. If $\left\|u_{0}\right\|_{V},\left\|w_{0}\right\|_{H_{0}^{1}},\left\|b_{0}\right\|_{V},\|f\|_{L^{\infty}\left([0, \infty) ; L^{2}(\Omega)\right)}$, and $\|g\|_{L^{\infty}\left([0, \infty) ; L^{2}(\Omega)\right)}$ are sufficiently small, then the solution $(u, w, b)$ of problem $(2.5)$ exists globally in time and satisfies $u, b \in C([0, \infty) ; V)$ and $w \in C\left([0, \infty) ; H_{0}^{1}(\Omega)\right)$. Moreover, for any $\theta>0$ there exists some finite positive constants $M$ and $C$ such that

$$
\begin{gathered}
\sup _{t \geq 0}\{\|\nabla u(t)\|,\|\nabla w(t)\|,\|\nabla b(t)\|\}=M, \\
\sup _{t \geq 0} e^{-\theta t} \int_{0}^{t} e^{\theta s}\left\{\left\|u_{t}(s)\right\|^{2}+j\left\|w_{t}(s)\right\|^{2}+\left\|b_{t}(s)\right\|^{2}\right\} d s \leq C, \\
\sup _{t \geq 0} e^{-\theta t} \int_{0}^{t} e^{\theta s}\left\{\|A u(s)\|^{2}+\|B w(s)\|^{2}+\|A b(s)\|^{2}\right\} d s \leq C .
\end{gathered}
$$

Also, the same kind of estimates hold uniformly in $k$ for the Galerkin approximations.

Proof. We will combine arguments used by Rojas-Medar [9] and Heywood and Rannacher [5]. We start by proving the boundness in time of $\|\nabla u(t)\|^{2}+j\|\nabla w(t)\|^{2}+$ $\|\nabla b(t)\|^{2}$.

From Rojas-Medar [9, page 11], we have the following differential equality:

$$
\frac{d}{d t} \xi(t)+\tau(t) \leq c \xi^{3}(t)+c \xi(t)+c\|f\|^{2}+c\|g\|^{2}
$$

where $\xi(t)=\|\nabla u(t)\|^{2}+j\|\nabla w(t)\|^{2}+\|\nabla b(t)\|^{2}, \tau(t)=c\left(\|A u(t)\|^{2}+\|A b(t)\|^{2}+\right.$ $\left.\|B w(t)\|^{2}\right)$.

Now, using the same arguments used by Heywood and Rannacher [5, page 283], for the Navier-Stokes equations, we can conclude that

$$
\sup _{t \geq 0} \xi(t) \equiv \sup _{t \geq 0}\left(\|\nabla u(t)\|^{2}+j\|\nabla w(t)\|^{2}+\|\nabla b(t)\|^{2}\right) \equiv C^{2} .
$$

Multiplying the inequality (3.4) by $e^{\theta t}, \theta>0$, and integrating in time from 0 to $t$, 
116 Magneto-micropolar fluid motion: global existence of strong solutions

we have

$$
\begin{aligned}
e^{\theta t}\left(\|\nabla u(t)\|^{2}\right. & \left.+j\|\nabla w(t)\|^{2}+\|\nabla b(t)\|^{2}\right) \\
& +c \int_{0}^{t} e^{\theta s}\left(\|A u(s)\|^{2}+\|A b(s)\|^{2}+\|B w(s)\|^{2}\right) d s \\
\leq & \|\nabla u(0)\|^{2}+j\|\nabla w(0)\|^{2}+\|\nabla b(0)\|^{2} \\
& +c \int_{0}^{t} e^{\theta s}\left[\|\nabla u(s)\|^{2}+j\|\nabla w(s)\|^{2}+\|\nabla b(s)\|^{2}\right]^{3} d s \\
& +(c+\theta) \int_{0}^{t} e^{\theta s}\left(\|\nabla u(s)\|^{2}+j\|\nabla w(s)\|^{2}+\|\nabla b(s)\|^{2}\right) d s \\
& +c\left(\sup _{t \geq 0}\|f(t)\|^{2}+\sup _{t \geq 0}\|g(t)\|^{2}\right) \int_{0}^{t} e^{\theta s} d s .
\end{aligned}
$$

Multiplying by $e^{-\theta t}$ and recalling that $\|\nabla u(t)\|,\|\nabla w(t)\|$, and $\|\nabla b(t)\|$ are uniformly bounded, we get that

$$
e^{-\theta t} \int_{0}^{t} e^{\theta s}\|A u(s)\|^{2} d s, \quad e^{-\theta t} \int_{0}^{t} e^{\theta s}\|B w(s)\|^{2} d s, \quad e^{-\theta t} \int_{0}^{t} e^{\theta s}\|A b(s)\|^{2} d s
$$

are also uniformly bounded.

Now, we proceed to prove the other stated estimates. They should be proved first for the approximations $\left(u^{k}, w^{k}, b^{k}\right)$ and then carried to $(u, w, b)$ in the limit. Since that is a standard procedure and the computations are exactly the same, to ease the notation, we will work directly with $(u, w, b)$ in the rest of the paper.

Also, we would like to mention that the technique of using exponentials as weight functions in time was inspired by Heywood and Rannacher [5]. Now, by taking $\varphi=u_{t}$ in (2.5a), $\phi=w_{t}$ in (2.5b), and $\psi=b_{t}$ in (2.5c), we obtain

$$
\begin{aligned}
& \left\|u_{t}\right\|^{2}=\chi\left(\operatorname{rot} w, u_{t}\right)+r\left(b \cdot \nabla b, u_{t}\right)-\left(u \cdot \nabla u, u_{t}\right)-(\mu+\chi)\left(A u, u_{t}\right)+\left(f, u_{t}\right), \\
& \frac{\alpha+\beta}{2} \frac{d}{d t}\|\operatorname{div} w\|^{2}+j\left\|w_{t}\right\|^{2} \\
& =\chi\left(\operatorname{rot} u, w_{t}\right)-2 \chi\left(w, w_{t}\right)-j\left(u \cdot \nabla w, w_{t}\right)-\gamma\left(B w, w_{t}\right)+\left(g, w_{t}\right), \\
& \left\|b_{t}\right\|^{2}=\left(b \cdot \nabla u, b_{t}\right)-\left(u \cdot \nabla b, b_{t}\right)-v\left(A b, b_{t}\right) .
\end{aligned}
$$

From this, we have

$$
\begin{aligned}
e^{-\theta t} \int_{0}^{t} e^{\theta s}\left\|u_{t}(s)\right\|^{2} d s \leq & c e^{-\theta t} \int_{0}^{t} e^{\theta s}\left(\|\nabla w(s)\|^{2}+\|A u(s)\|^{2}\right) d s+c e^{-\theta t} \int_{0}^{t} e^{\theta s} d s \\
& +c e^{-\theta t} \int_{0}^{t} e^{\theta s}\left(\|u(s) \cdot \nabla u(s)\|^{2}+\|b(s) \cdot \nabla b(s)\|^{2}\right) d s,
\end{aligned}
$$




$$
\begin{aligned}
(\alpha+\beta)\|\operatorname{div} w(t)\|^{2}+ & j e^{-\theta t} \int_{0}^{t} e^{\theta s}\left\|w_{t}(s)\right\|^{2} d s \\
\leq & (\alpha+\beta) e^{-\theta t}\left\|\operatorname{div} w_{0}\right\|^{2}+(\alpha+\beta) \theta e^{-\theta t} \int_{0}^{t} e^{\theta s}\|\operatorname{div} w(s)\|^{2} d s \\
& +c e^{-\theta t} \int_{0}^{t} e^{\theta s}\left(\|\nabla u(s)\|^{2}+\|\nabla w(s)\|^{2}+\|B w(s)\|^{2}\right) d s \\
& +c e^{-\theta t} \int_{0}^{t} e^{\theta s}\|u(s) \cdot \nabla w(s)\|^{2} d s+c e^{-\theta t} \int_{0}^{t} e^{\theta s} d s \\
e^{-\theta t} \int_{0}^{t} e^{\theta s}\left\|b_{t}(s)\right\|^{2} d s \leq & c e^{-\theta t} \int_{0}^{t} e^{\theta s}\|A b(s)\|^{2} d s \\
& +c e^{-\theta t} \int_{0}^{t} e^{\theta s}\left(\|u(s) \cdot \nabla b(s)\|^{2}+\|b(s) \cdot \nabla u(s)\|^{2}\right) d s
\end{aligned}
$$

Now, bearing in mind (3.1) and the Sobolev embedding $H^{2}(\Omega) \hookrightarrow L^{\infty}(\Omega)$, we obtain the following estimate

$$
\|u \cdot \nabla u\|^{2} \leq\|u\|_{L^{\infty}}^{2}\|\nabla u\|^{2} \leq c M^{2}\|A u\|^{2} .
$$

Consequently,

$$
e^{-\theta t} \int_{0}^{t} e^{\theta s}\|u(s) \cdot \nabla u(s)\|^{2} d s \leq c M^{2} e^{-\theta t} \int_{0}^{t} e^{\theta s}\|A u(s)\|^{2} d s \leq C M^{2},
$$

thanks to the estimate (3.3). The other terms in (3.9), (3.10), and (3.11) are also estimates, moreover using the estimate (3.1) and (3.3), we obtain the desired result. Now, we observe that the previous estimates hold true for $\theta \geq 0$ if we are considering finite time intervals $[0, T], 0<T<+\infty$ (with the suprema obviously depending on $T$ ). This comes from the way that the proofs were done.

Thus, in a finite interval $[0, T]$, we can take the last estimates with $\theta=0$.

Remark 3.2. As in the end of the previous proof, we observe that all these estimates hold true for $\theta=0$ on the time interval $[0, \infty)$ if we also include in the hypothesis $f, g \in L^{2}\left([0, \infty) ; L^{2}(\Omega)\right)$.

\section{More regular solution}

The following result is the analogue of Theorem 2.4.

THEOREM 4.1. In addition to the assumptions of Theorem 3.1, assume that $u_{0}, b_{0} \in V \cap$ $H^{2}(\Omega), w_{0} \in H_{0}^{1}(\Omega) \cap H^{2}(\Omega)$, and $f_{t}, g_{t} \in L^{\infty}\left([0, \infty) ; L^{2}(\Omega)\right)$. Then, the solution obtained in Theorem 3.1 satisfies

$$
\begin{gathered}
u, b \in C\left([0, \infty) ; V \cap H^{2}(\Omega)\right) \cap C^{1}([0, \infty) ; H), \\
w \in C\left([0, \infty) ; H_{0}^{1}(\Omega) \cap H^{2}(\Omega)\right) \cap C^{1}\left([0, \infty) ; L^{2}(\Omega)\right) .
\end{gathered}
$$


118 Magneto-micropolar fluid motion: global existence of strong solutions

Moreover, for any $\theta>0$ there exists one positive constant $C$ such that

$$
\begin{gathered}
\sup _{t \geq 0}\left\{\left\|u_{t}(t)\right\|,\left\|b_{t}(t)\right\|,\left\|w_{t}(t)\right\|\right\} \leq C, \\
\sup _{t \geq 0}\{\|A u(t)\|,\|A b(t)\|,\|B w(t)\|\} \leq C, \\
\sup _{t \geq 0} e^{-\theta t} \int_{0}^{t} e^{\theta s}\left(\left\|\nabla u_{t}(s)\right\|^{2}+\left\|\nabla w_{t}(s)\right\|^{2}+\left\|\nabla b_{t}(s)\right\|^{2}\right) d s \leq C, \\
\sup _{t \geq 0} e^{-\theta t} \int_{0}^{t} e^{\theta s}\left(\left\|u_{t t}(s)\right\|_{V^{*}}^{2}+\left\|w_{t t}(s)\right\|_{H^{-1}}^{2}+\left\|b_{t t}(s)\right\|_{V^{*}}^{2}\right) d s \leq C .
\end{gathered}
$$

Also, the same kind of estimates hold uniformly in $k$ for the Galerkin approximations.

Proof. We will need further estimates for $u, w, b$ (actually $u^{k}, w^{k}$, and $b^{k}$ ). To this end, we differentiate (2.5a), (2.5b), and (2.5c) with respect to $t$ and set $\varphi=u_{t}, \phi=w_{t}$, and $\psi=r b_{t}$ (actually $\varphi=u_{t}^{k}, \phi=w_{t}^{k}$, and $\psi=r b_{t}^{k}$ ). We are left with

$$
\begin{gathered}
\frac{1}{2} \frac{d}{d t}\left\|u_{t}\right\|^{2}+(\mu+\chi)\left\|\nabla u_{t}\right\|^{2}= \\
-\left(\operatorname{rot} w_{t}, u_{t}\right)+r\left(b_{t} \cdot \nabla b, u_{t}\right)+r\left(b \cdot \nabla b_{t}, u_{t}\right) \\
\left.-u_{t}\right)+\left(f_{t}, u_{t}\right), \\
\frac{j}{2} \frac{d}{d t}\left\|w_{t}\right\|^{2}+\gamma\left\|\nabla w_{t}\right\|^{2}+(\alpha+\beta)\left\|\operatorname{div} w_{t}\right\|^{2}+2 \chi\left\|w_{t}\right\|^{2} \\
=\chi\left(\operatorname{rot} u_{t}, w_{t}\right)-j\left(u_{t} \cdot \nabla w, w_{t}\right)+\left(g_{t}, w_{t}\right), \\
\frac{r}{2} \frac{d}{d t}\left\|b_{t}\right\|^{2}+r v\left\|\nabla b_{t}\right\|^{2}=r\left(b_{t} \cdot \nabla u, b_{t}\right)+r\left(b \cdot \nabla u_{t}, b_{t}\right)-r\left(u_{t} \cdot \nabla b, b_{t}\right),
\end{gathered}
$$

since $\left(u \cdot \nabla u_{t}, u_{t}\right)=\left(u \cdot \nabla w_{t}, w_{t}\right)=\left(u \cdot \nabla b_{t}, b_{t}\right)=0$.

We observe that

$$
\begin{gathered}
\left|\chi\left(\operatorname{rot} w_{t}, u_{t}\right)\right| \leq c\left\|u_{t}\right\|^{2}+\frac{\gamma}{6}\left\|\nabla w_{t}\right\|^{2}, \\
\left|\chi\left(\operatorname{rot} u_{t}, w_{t}\right)\right| \leq c\left\|w_{t}\right\|^{2}+\frac{(\mu+\chi)}{12}\left\|\nabla u_{t}\right\|^{2}, \\
\left|\left(f_{t}, u_{t}\right)\right| \leq c\left\|f_{t}\right\|^{2}+\frac{(u+\chi)}{12}\left\|\nabla u_{t}\right\|^{2}, \\
\left|\left(g_{t}, w_{t}\right)\right| \leq c\left\|g_{t}\right\|^{2}+\frac{\gamma}{6}\left\|\nabla w_{t}\right\|^{2} .
\end{gathered}
$$

Now, by using the following Sobolev type inequality $\|\varphi\|_{L^{4}} \leq c\|\varphi\|^{1 / 4}\|\nabla \varphi\|^{3 / 4}$, 
we have

$$
\begin{aligned}
\left|\left(u_{t} \cdot \nabla u, u_{t}\right)\right| & \leq\|\nabla u\|\left\|u_{t}\right\|_{L^{4}}^{2} \leq c\|\nabla u\|\left\|u_{t}\right\|^{1 / 2}\left\|\nabla u_{t}\right\|^{3 / 2} \\
& \leq c\|\nabla u\|^{4}\left\|u_{t}\right\|^{2}+\frac{(\mu+\chi)}{12}\left\|\nabla u_{t}\right\|^{2}, \\
\left|r\left(b_{t} \cdot \nabla b, u_{t}\right)\right| & \leq r\left\|b_{t}\right\|_{L^{4}}\|\nabla b\|\left\|u_{t}\right\|_{L^{4}} \leq c\left\|b_{t}\right\|_{L^{4}}\|\nabla b\|\left\|\nabla u_{t}\right\| \\
& \leq c\|\nabla b\|^{2}\left\|b_{t}\right\|^{1 / 2}\left\|\nabla b_{t}\right\|^{3 / 2}+\frac{(\mu+\chi)}{12}\left\|\nabla u_{t}\right\|^{2} \\
& \leq c\|\nabla b\|^{8}\left\|b_{t}\right\|^{2}+\frac{r v}{6}\left\|\nabla b_{t}\right\|^{2}+\frac{(\mu+\chi)}{12}\left\|\nabla u_{t}\right\|^{2} .
\end{aligned}
$$

Analogously, we can prove

$$
\begin{aligned}
\left|r\left(u_{t} \cdot \nabla b, b_{t}\right)\right| & \leq c\|\nabla b\|^{8}\left\|b_{t}\right\|^{2}+\frac{r v}{6}\left\|\nabla b_{t}\right\|^{2}+\frac{(\mu+\chi)}{12}\left\|\nabla u_{t}\right\|^{2}, \\
\left|r\left(b_{t} \cdot \nabla u, b_{t}\right)\right| & \leq c\|\nabla u\|^{4}\left\|b_{t}\right\|^{2}+\frac{r v}{6}\left\|\nabla b_{t}\right\|^{2}, \\
\left|j\left(u_{t} \cdot \nabla w, w_{t}\right)\right| & \leq c\|\nabla w\|^{8}\left\|w_{t}\right\|^{2}+\frac{\gamma}{6}\left\|\nabla w_{t}\right\|^{2}+\frac{(\mu+\chi)}{12}\left\|\nabla u_{t}\right\|^{2} .
\end{aligned}
$$

Adding the inequalities (4.6), (4.7), and (4.8), observing that $r\left(b \cdot \nabla b_{t}, u_{t}\right)+r(b$. $\left.\nabla u_{t}, b_{t}\right)=0$ and using the above estimates, we are left with the following differential inequality,

$$
\begin{aligned}
& \frac{d}{d t}\left(\left\|u_{t}\right\|^{2}+j\left\|w_{t}\right\|^{2}+r\left\|b_{t}\right\|^{2}\right)+c_{1}\left(\left\|\nabla u_{t}\right\|^{2}+\left\|\nabla w_{t}\right\|^{2}+\left\|\nabla b_{t}\right\|^{2}\right) \\
& \leq c(M)\left(\left\|u_{t}\right\|^{2}+j\left\|w_{t}\right\|^{2}+r\left\|b_{t}\right\|^{2}\right)+C\left(\left\|f_{t}\right\|^{2}+\left\|g_{t}\right\|^{2}\right),
\end{aligned}
$$

where $c_{1}=\min \{(\mu+\chi), \gamma, r \nu\}>0$.

By multiplying the above inequality for $e^{\theta t}$ and integrating in time the resulting inequality from 0 to $t$, we obtain

$$
\begin{aligned}
e^{\theta t}\left(\left\|u_{t}(t)\right\|^{2}+j\left\|w_{t}(t)\right\|^{2}+r\left\|b_{t}(t)\right\|^{2}\right) \\
\quad+c_{1} \int_{0}^{t} e^{\theta s}\left(\left\|\nabla u_{t}(s)\right\|^{2}+\left\|\nabla w_{t}(s)\right\|^{2}+\left\|\nabla b_{t}(s)\right\|^{2}\right) d s \\
\leq c(M) \int_{0}^{t} e^{\theta s}\left(\left\|u_{t}(s)\right\|^{2}+j\left\|w_{t}(s)\right\|^{2}+r\left\|b_{t}(s)\right\|^{2}\right) d s \\
\quad+c \int_{0}^{t} e^{\theta s}\left(\left\|f_{t}(s)\right\|^{2}+\left\|g_{t}(s)\right\|^{2}\right) d s+\left\|u_{t}(0)\right\|^{2}+j\left\|w_{t}(0)\right\|^{2} \\
\quad+r\left\|b_{t}(0)\right\|^{2}+\theta \int_{0}^{t} e^{\theta s}\left(\left\|u_{t}(s)\right\|^{2}+j\left\|w_{t}(s)\right\|^{2}+r\left\|b_{t}(s)\right\|^{2}\right) d s .
\end{aligned}
$$


120 Magneto-micropolar fluid motion: global existence of strong solutions

By multiplying the above inequality by $e^{-\theta t}$, we get

$$
\begin{aligned}
\left\|u_{t}(t)\right\|^{2} & +j\left\|w_{t}(t)\right\|^{2}+r\left\|b_{t}(t)\right\|^{2} \\
& +c_{1} e^{-\theta t} \int_{0}^{t} e^{\theta s}\left(\left\|\nabla u_{t}(s)\right\|^{2}+\left\|\nabla w_{t}(s)\right\|^{2}+\left\|\nabla b_{t}(s)\right\|^{2}\right) d s \\
\leq & (\theta+c(M)) e^{-\theta t} \int_{0}^{t} e^{\theta s}\left(\left\|u_{t}(s)\right\|^{2}+j\left\|w_{t}(s)\right\|^{2}+r\left\|b_{t}(s)\right\|^{2}\right) d s \\
& +c e^{-\theta t} \int_{0}^{t} e^{\theta s}\left(\left\|f_{t}(s)\right\|^{2}+\left\|g_{t}(s)\right\|^{2}\right) d s \\
& +e^{-\theta t}\left(\left\|u_{t}(0)\right\|^{2}+j\left\|w_{t}(0)\right\|^{2}+r\left\|b_{t}(0)\right\|^{2}\right) \\
\leq & C+e^{-\theta t}\left(\left\|u_{t}(0)\right\|^{2}+j\left\|w_{t}(0)\right\|^{2}+r\left\|b_{t}(0)\right\|^{2}\right),
\end{aligned}
$$

thanks to the previous estimates and our hypotheses on $f_{t}$ and $g_{t}$. So, it is enough to find estimates for $\left\|u_{t}(0)\right\|^{2},\left\|w_{t}(0)\right\|^{2}$, and $\left\|b_{t}(0)\right\|^{2}$ (actually, $\left\|u_{t}^{k}(0)\right\|^{2},\left\|w_{t}^{k}(0)\right\|^{2}$, and $\left.\left\|b_{t}^{k}(0)\right\|^{2}\right)$.

For this, recall that $u_{0}, b_{0}\left(u_{0}^{k}, b_{0}^{k}\right) \in V \cap H^{2}(\Omega)$, and $w_{0}\left(w_{0}^{k}\right) \in H_{0}^{1}(\Omega) \cap H^{2}(\Omega)$. Consequently, by setting $\varphi=u_{t}, \phi=w_{t}$, and $\psi=b_{t}$ in (2.5a), (2.5b), (2.5c), respectively, (actually $\varphi=u_{t}^{k}, \phi=w_{t}^{k}$, and $\psi=b_{t}^{k}$ in $(2.8 \mathrm{a}),(2.8 \mathrm{~b}),(2.8 \mathrm{c})$, respectively), we have

$$
\begin{aligned}
\left\|u_{t}\right\|^{2}= & \left(f, u_{t}\right)+\chi\left(\operatorname{rot} w, u_{t}\right)+r\left(b \cdot \nabla b, u_{t}\right)-(\mu+\chi)\left(A u, u_{t}\right)-\left(u \cdot \nabla u, u_{t}\right), \\
j\left\|w_{t}\right\|^{2}= & \left(g, w_{t}\right)+\chi\left(\operatorname{rot} u, w_{t}\right)-\gamma\left(B w, w_{t}\right)-2 \chi\left(w, w_{t}\right) \\
& +(\alpha+\beta)\left(\nabla \operatorname{div} w, w_{t}\right)-j\left(u \cdot \nabla w, w_{t}\right), \\
\left\|b_{t}\right\|^{2}= & \left(b \cdot \nabla u, b_{t}\right)-\left(u \cdot \nabla b, b_{t}\right)-v\left(A b, b_{t}\right)
\end{aligned}
$$

the above inequalities imply,

$$
\begin{aligned}
\left\|u_{t}(0)\right\| \leq & \|f(0)\|+c \chi\left\|\nabla w_{0}\right\|+c r\left\|A b_{0}\right\|\left\|\nabla b_{0}\right\| \\
& +c(\mu+\chi)\left\|A u_{0}\right\|+c\left\|A u_{0}\right\|\left\|\nabla u_{0}\right\| \leq C, \\
j\left\|w_{t}(0)\right\| \leq & \|g(0)\|+c \chi\left\|\nabla u_{0}\right\|+\gamma\left\|B w_{0}\right\|+2 \chi\left\|w_{0}\right\| \\
& +(\alpha+\beta)\left\|\nabla \operatorname{div} w_{0}\right\|+c j\left\|A u_{0}\right\|\left\|\nabla w_{0}\right\| \leq C, \\
\left\|b_{t}(0)\right\| \leq & c\left\|A b_{0}\right\|\left\|\nabla u_{0}\right\|+c\left\|A u_{0}\right\|\left\|\nabla b_{0}\right\|+v\left\|A b_{0}\right\| \leq C .
\end{aligned}
$$

Now, by taking $\varphi=A u$ and $\psi=A b$ in (2.5a), (2.5c), respectively (actually $\varphi=A u^{k}$ and $\psi=A b^{k}$ in (2.8a), (2.8c), respectively), we get

$$
\begin{gathered}
(\mu+\chi)\|A u\|^{2} \leq C\left(\|f\|^{2}+\left\|u_{t}\right\|^{2}+\|\nabla w\|^{2}+\|u \cdot \nabla u\|^{2}+\|b \cdot \nabla b\|^{2}\right), \\
v\|A b\|^{2} \leq C\left(\left\|b_{t}\right\|^{2}+\|u \cdot \nabla b\|^{2}+\|b \cdot \nabla u\|^{2}\right) .
\end{gathered}
$$


We observe that

$$
\begin{aligned}
\|u \cdot \nabla u\|^{2} & \leq\|u\|_{L^{4}}^{2}\|\nabla u\|_{L^{4}}^{2} \leq c\|\nabla u\|^{2}\left(\|\nabla u\|^{1 / 2}\|A u\|^{3 / 2}\right) \\
& \leq c\|\nabla u\|^{5 / 2}\|A u\|^{3 / 2} \leq c\|\nabla u\|^{10}+\frac{1}{4}(\mu+\chi)\|A u\|^{2} .
\end{aligned}
$$

Analogously as above, we obtain

$$
\begin{gathered}
\|b \cdot \nabla b\|^{2} \leq C\|\nabla b\|^{10}+\frac{1}{4} v\|A b\|^{2}, \\
\|u \cdot \nabla b\|^{2} \leq C\|\nabla u\|^{8}\|\nabla b\|^{2}+\frac{1}{4} v\|A b\|^{2}, \\
\|b \cdot \nabla u\|^{2} \leq C\|\nabla b\|^{8}\|\nabla u\|^{2}+\frac{1}{4}(\mu+\chi)\|A u\|^{2} .
\end{gathered}
$$

Now, by adding the inequalities (4.17) and (4.18) and by using the estimates (4.19), (4.20), (4.21), and (4.22) together with the estimates (3.1) and (4.2), we obtain

$$
\sup _{t \geq 0}\|A u(t)\|^{2} \leq C, \quad \sup _{t \geq 0}\|A b(t)\|^{2} \leq C .
$$

Taking $\phi=B w$ in (2.5b) and using the fact that the operator

$$
L w=\gamma \Delta w+(\alpha+\beta) \nabla \operatorname{div} w
$$

is a strongly elliptic operator, this implies that

$$
(L w, \Delta w) \geq \gamma\|\Delta w\|^{2}-C_{0}\|\nabla w\|^{2},
$$

where $C_{0}>0$ depend on $\gamma, \alpha+\beta$ and $\partial \Omega$, we have for any $\sigma>0$

$$
\begin{aligned}
\gamma\|B w\|^{2} \leq & c_{\sigma}\left(C_{0}\|\nabla w\|^{2}+j\left\|w_{t}\right\|^{2}+c j\|A u\|^{2}\|\nabla w\|^{2}+2 \chi\|w\|^{2}+\chi\|\nabla u\|^{2}\right) \\
& +c_{\sigma}\|g\|^{2}+5 \sigma\|B w\|^{2} .
\end{aligned}
$$

By taking $\sigma=(1 / 10) \gamma$, we obtain

$$
\gamma\|B w\|^{2} \leq c M+c M\|A u\|^{2}+c\left\|w_{t}\right\|^{2}+c\|g\|^{2} .
$$

Consequently, using the estimates (4.2) and (4.23), we obtain

$$
\sup _{t \geq 0}\|B w\|^{2} \leq C
$$

We differentiate (2.5a) (actually (2.7a)) with respect to $t$, and we obtain

$$
\begin{aligned}
u_{t t}= & \chi P\left(\operatorname{rot} w_{t}\right)+r P\left(b_{t} \cdot \nabla b\right)+r P\left(b \cdot \nabla b_{t}\right)-(\mu+\chi) A u_{t} \\
& -P\left(u_{t} \cdot \nabla u\right)-P\left(u \cdot \nabla u_{t}\right)+P f_{t} \equiv h .
\end{aligned}
$$


122 Magneto-micropolar fluid motion: global existence of strong solutions

Consequently

$$
e^{-\theta t} \int_{0}^{t} e^{\theta s}\left\|u_{t t}(s)\right\|_{V^{*}}^{2} d s \leq e^{-\theta t} \int_{0}^{t} e^{\theta s}\|h(s)\|_{V^{*}}^{2} d s
$$

this is sufficient to estimate the right-hand side. To do this, we observe that

$$
\left\|A u_{t}\right\|_{V^{*}}=\sup _{\|v\|_{V} \leq 1}\left|\left(A u_{t}, v\right)\right|=\sup _{\|v\|_{V} \leq 1}\left|\left(\nabla u_{t}, \nabla v\right)\right| \leq\left\|\nabla u_{t}\right\|
$$

then, for all $t \geq 0$

$$
e^{-\theta t} \int_{0}^{t} e^{\theta s}\left\|A u_{t}(s)\right\|_{V^{*}}^{2} d s \leq e^{-\theta t} \int_{0}^{t} e^{\theta s}\left\|\nabla u_{t}(s)\right\|^{2} d s \leq C
$$

thanks to the estimate (4.4). Also, we have

$$
\left\|P\left(\operatorname{rot} w_{t}\right)\right\|_{V^{*}}=\sup _{\|v\|_{V} \leq 1}\left|\left(\operatorname{rot} w_{t}, v\right)\right| \leq c\left\|\nabla w_{t}\right\| .
$$

Consequently, for all $t \geq 0$,

$$
e^{-\theta t} \int_{0}^{t} e^{\theta s}\left\|P\left(\operatorname{rot} w_{t}(s)\right)\right\|_{V^{*}}^{2} d s \leq c e^{-\theta t} \int_{0}^{t} e^{\theta s}\left\|\nabla w_{t}(s)\right\|^{2} d s \leq C
$$

thanks to the estimate (4.4). Finally, we have

$$
\left\|P\left(u_{t} \cdot \nabla u\right)\right\|_{V^{*}}=\sup _{\|v\|_{V} \leq 1}\left|\left(u_{t} \cdot \nabla u, v\right)\right| \leq c \sup _{\|v\|_{V} \leq 1}\left\|\nabla u_{t}\right\|\|\nabla u\|\|\nabla v\| \leq c M\left\|\nabla u_{t}\right\|,
$$

this implies that, for all $t \geq 0$,

$$
e^{-\theta t} \int_{0}^{t} e^{\theta s}\left\|P\left(u_{t}(s) \cdot \nabla u(s)\right)\right\|_{V^{*}}^{2} d s \leq c M^{2} e^{-\theta t} \int_{0}^{t} e^{\theta s}\left\|\nabla u_{t}(s)\right\|^{2} d s \leq C .
$$

The other terms in (4.29) are analogously estimated. So, we obtain

$$
\sup _{t \geq 0} e^{-\theta t} \int_{0}^{t} e^{\theta s}\left\|u_{t t}(s)\right\|_{V^{*}}^{2} d s \leq \sup _{t \geq 0} e^{-\theta t} \int_{0}^{t} e^{\theta s}\|h(s)\|_{V^{*}}^{2} d s \leq C .
$$

The results for $b_{t t}$ and $w_{t t}$ are quite similar. To finish the proof we have to show the continuity of $u(t), w(t)$, and $b(t)$ in the $H^{2}$-norm. We observe that

$$
\begin{aligned}
(\mu+\chi) A u(t) & =\chi P(\operatorname{rot} w(t))+P f(t)+r P(b(t) \cdot \nabla b(t))-P(u(t) \cdot \nabla u(t))-u_{t}(t) \\
& \equiv L(t) .
\end{aligned}
$$

Recalling that $w \in C\left([0, \infty) ; H_{0}^{1}(\Omega)\right)$ then $\operatorname{rot} w \in C\left([0, \infty) ; L^{2}(\Omega)\right)$ and consequently, $P(\operatorname{rot} w) \in C([0, \infty) ; H)$. Since $f, f_{t} \in L^{\infty}\left([0, \infty) ; L^{2}(\Omega)\right)$, by interpolation we have that $f \in C\left([0, \infty) ; L^{2}(\Omega)\right)$ and consequently, $P f \in C([0, \infty) ; H)$. Also, $u \in C([0, \infty)$; 
$V)$ and the estimate $\|A u\| \leq C$ implies that $u \cdot \nabla u \in C\left([0, \infty) ; L^{2}(\Omega)\right)$. In fact, we have

$$
\begin{aligned}
\| u(t) \cdot \nabla & u(t)-u\left(t_{0}\right) \cdot \nabla u\left(t_{0}\right) \| \\
& \leq\left\|\left(u(t)-u\left(t_{0}\right)\right) \cdot \nabla u(t)\right\|+\left\|u\left(t_{0}\right) \cdot \nabla\left(u(t)-u\left(t_{0}\right)\right)\right\| \\
& \leq C\|A u(t)\|\left\|\nabla u(t)-\nabla u\left(t_{0}\right)\right\|+C\left\|A u\left(t_{0}\right)\right\|\left\|\nabla\left(u(t)-u\left(t_{0}\right)\right)\right\| \\
& \leq C\left\|\nabla\left(u(t)-u\left(t_{0}\right)\right)\right\| \longrightarrow 0,
\end{aligned}
$$

as $t \rightarrow t_{0}$. Finally, we conclude $P(u \cdot \nabla u) \in C([0, \infty) ; H)$. Analogously, we obtain $P(b \cdot \nabla b) \in C([0, \infty) ; H)$, and since $u_{t} \in C([0, \infty) ; H)$, we conclude that $L \in$ $C([0, \infty) ; H)$. Consequently, $A u \in C([0, \infty) ; H)$, and this implies that $u \in C([0, \infty)$; $D(A))$. Analogously, we prove the continuity of $w$ and $b$.

\section{Results on the pressure}

In a standard way we can obtain information on the pressure. In fact, we have the following proposition.

Proposition 5.1. Under the hypotheses of Theorem 3.1, there exists a unique function $p^{*} \in L_{\mathrm{Loc}}^{2}\left([0, \infty) ; H^{1}(\Omega) / \mathbb{R}\right)$ such that by taking $p=p^{*}-(1 / 2) r b \cdot b,(u, w, b, p)$ is the solution of (1.1), (1.2), and (1.3) and satisfies for any $\theta>0$

$$
\sup _{t \geq 0} e^{-\theta t} \int_{0}^{t} e^{\theta s}\|p(s)\|_{H^{1}(\Omega) / \mathbb{R}}^{2} d s \leq C,
$$

under the hypotheses of Theorem 4.1,

$$
p^{*} \in L^{\infty}\left([0, \infty) ; H^{1}(\Omega) / \mathbb{R}\right) \cap C\left([0, \infty) ; L^{2}(\Omega) / \mathbb{R}\right),
$$

and $p=p^{*}-(1 / 2) r b \cdot b$, satisfies

$$
\sup _{t \geq 0}\|p(t)\|_{H^{1}(\Omega) / \mathbb{R}} \leq C .
$$

Proof. We observe that (1.1) is equivalent to

$$
(\mu+\chi) A u=P(F),
$$

where $F=f+\chi \operatorname{rot} w+r b \cdot \nabla b-u \cdot \nabla u-u_{t}$.

Now, we observe that under the hypotheses of Theorem 3.1 (respectively, of Theorem 4.1 ), we have $F \in L_{\text {Loc }}^{2}\left([0, \infty) ; L^{2}(\Omega)\right)$ (respectively, $F \in L^{\infty}\left([0, \infty) ; L^{2}(\Omega)\right)$ ).

Therefore, Amrouche and Girault's results [2] imply that there exists a unique $p^{*} \in L_{\text {Loc }}^{2}\left([0, \infty) ; H^{1}(\Omega) / \mathbb{R}\right)$ (respectively, $p^{*} \in L^{\infty}\left([0, \infty) ; H^{1}(\Omega) / \mathbb{R}\right) \cap C([0, \infty) ;$ $\left.L^{2}(\Omega) / \mathbb{R}\right)$ ) such that

$$
-(\mu+\chi) \Delta u+\nabla p^{*}=F, \quad \operatorname{div} u=0,\left.\quad u\right|_{\partial \Omega}=0 .
$$

Now, it is enough to take $p=p^{*}-(r / 2) b \cdot b$ and the proposition is proved. Estimates (5.1) (respectively (5.3)) follows easily from the previous estimates and the estimates given in the above section. This completes the proof of the proposition. 
124 Magneto-micropolar fluid motion: global existence of strong solutions

\section{Global existence with exponential decay in time of the external forces}

The analogue to Theorem 3.1 is the following theorem.

THEOREM 6.1. Under the hypotheses of Theorem 3.1, we assume that for some $\bar{\gamma}>0$, $e^{\bar{\gamma} t} f, e^{\bar{\gamma} t} g \in L^{\infty}\left([0, \infty) ; L^{2}(\Omega)\right)$ with $\left\|e^{\bar{\gamma} t} f\right\|_{L^{\infty}\left([0, \infty) ; L^{2}(\Omega)\right)}$ and $\left\|e^{\bar{\gamma} t} g\right\|_{L^{\infty}\left([0, \infty) ; L^{2}(\Omega)\right)}$ sufficiently small. Then, the unique global strong solution $(u, w, b)$ of the problem $(2.5)$ given by Theorem 3.1, satisfies

$$
u, b \in L^{2}([0, \infty) ; D(A)) \quad \text { and } \quad w \in L^{2}([0, \infty) ; D(B)) .
$$

Moreover, there exists a positive constant $\gamma^{*} \leq \bar{\gamma}$ such that $0 \leq \theta<\gamma^{*}$, are true

$$
\begin{gathered}
\sup _{t \geq 0} e^{\gamma^{*} t}\left(\|\nabla u(t)\|^{2}+\|\nabla w(t)\|^{2}+\|\nabla b(t)\|^{2}\right) \leq C, \\
\sup _{t \geq 0} \int_{0}^{t} e^{\theta \tau}\left(\|\nabla u(\tau)\|^{2}+\|\nabla w(\tau)\|^{2}+\|\nabla b(\tau)\|^{2}\right) d \tau \leq C, \\
\sup _{t \geq 0} \int_{0}^{t} e^{\theta \tau}\left(\|A u(\tau)\|^{2}+\|B w(\tau)\|^{2}+\|A b(\tau)\|^{2}\right) d \tau \leq C, \\
\sup _{t \geq 0} \int_{0}^{t} e^{\theta \tau}\left(\left\|u_{t}(\tau)\right\|^{2}+\left\|w_{t}(\tau)\right\|^{2}+\left\|b_{t}(\tau)\right\|^{2}\right) d \tau \leq C,
\end{gathered}
$$

where $C$ is a generic constant independent of $t$. Also, the same kind of estimates hold uniformly in $k$ for the Galerkin approximations.

The following result is the analogue of Theorem 4.1 .

TheOREM 6.2. In addition to the assumptions of Theorems 4.1 and 6.1, assume that $e^{\bar{\gamma} t} f_{t}, e^{\bar{\gamma} t} g_{t} \in L^{\infty}\left([0, \infty) ; L^{2}(\Omega)\right)$. Then, the unique global strong solution $(u, w, b)$ given by Theorem 4.1, for the same $\gamma^{*}$ and $\theta$ of Theorem 6.1, satisfies the following estimates:

$$
\begin{gathered}
\sup _{t \geq 0} e^{\theta t}\left(\left\|u_{t}(t)\right\|^{2}+\left\|w_{t}(t)\right\|^{2}+\left\|b_{t}(t)\right\|^{2}\right) \leq C, \\
\sup _{t \geq 0} \int_{0}^{t} e^{\theta s}\left(\left\|\nabla u_{t}(s)\right\|^{2}+\left\|\nabla w_{t}(s)\right\|^{2}+\left\|\nabla b_{t}(s)\right\|^{2}\right) d s \leq C, \\
\sup _{t \geq 0} e^{\theta t}\left(\|A u(t)\|^{2}+\|B w(t)\|^{2}+\|A b(t)\|^{2}\right) \leq C, \\
\sup _{t \geq 0} \int_{0}^{t} e^{\theta s}\left(\left\|u_{t t}(s)\right\|_{V^{*}}^{2}+\left\|w_{t t}(s)\right\|_{H^{-1}}^{2}+\left\|b_{t t}(s)\right\|_{V^{*}}^{2}\right) d s \leq C, \\
\sup _{t \geq 0} \sigma(t)\left(\left\|\nabla u_{t}(t)\right\|^{2}+\left\|\nabla w_{t}(t)\right\|^{2}+\left\|\nabla b_{t}(t)\right\|^{2}\right) \leq C, \\
\sup _{t \geq 0} \int_{0}^{t} \sigma(s)\left(\left\|u_{t t}(s)\right\|^{2}+\left\|w_{t t}(s)\right\|^{2}+\left\|b_{t t}(s)\right\|^{2}\right) d s \leq C,
\end{gathered}
$$

where $\sigma(t)=\min \{1, t\} e^{\theta t}$ and $C$ is a generic constant independent of $t$. Also, the same kind of estimates hold uniformly in $k$ for the Galerkin approximations. 


\section{Acknowledgement}

This research was supported by CAPES-Brazil and in part by a grant 300116/93-4(RN), CNPq-Brazil and FAPESP-Brazil under grant 1997/3711-0.

\section{References}

[1] G. Ahmadi and M. Shahinpoor, Universal stability of magneto-micropolar fluid motions, Internat. J. Engrg. Sci. 12 (1974), 657-663. MR 56\#1919. Zbl 284.76009.

[2] C. Amrouche and V. Girault, On the existence and regularity of the solution of Stokes problem in arbitrary dimension, Proc. Japan Acad. Ser. A Math. Sci. 67 (1991), no. 5, 171-175. MR 92i:35098. Zbl 752.35047.

[3] L. Cattabriga, Su un problema al contorno relativo al sistema di equazioni di Stokes, Rend. Sem. Mat. Univ. Padova 31 (1961), 308-340 (Italian). MR 25\#2334. Zbl 116.18002.

[4] G. P. Galdi and S. Rionero, A note on the existence and uniqueness of solutions of the micropolar fluid equations, Internat. J. Engrg. Sci. 15 (1977), no. 2, 105-108. MR 57\#6899. Zbl 351.76006.

[5] J. G. Heywood and R. Rannacher, Finite element approximation of the nonstationary NavierStokes problem. I. Regularity of solutions and second-order error estimates for spatial discretization, SIAM J. Numer. Anal. 19 (1982), no. 2, 275-311. MR 83d:65260. Zbl 487.76035.

[6] G. Lukaszewicz, On nonstationary flows of asymmetric fluids, Rend. Accad. Naz. Sci. XL Mem. Mat. (5) 12 (1988), no. 1, 83-97. MR 90f:35165. Zbl 668.76045.

[7] On the existence, uniqueness and asymptotic properties for solutions of flows of asymmetric fluids, Rend. Accad. Naz. Sci. XL Mem. Mat. (5) 13 (1989), no. 1, 105-120. MR 91d:35174. Zbl 692.76020.

[8] M. Padula and R. Russo, A uniqueness theorem for micropolar fluid motions in unbounded regions, Boll. Un. Mat. Ital. A (5) 13 (1976), no. 3, 660-666. MR 54\#14552. Zbl 355.76007.

[9] M. A. Rojas-Medar, Magneto-micropolar fluid motion: Existence and uniqueness of strong solution, Math. Nachr. 188 (1997), 301-319. MR 99h:76094. Zbl 893.76006.

[10] R. Temam, Navier-Stokes Equations. Theory and Numerical Analysis, revised ed., Studies in Mathematics and its Applications, vol. 2, North-Holland Publishing Co., Amsterdam, New York, Oxford, 1979. MR 82b:35133. Zbl 426.35003.

Elva E. Ortega-Torres: Universidad de Antofagasta, Departamento de Matemáticas, Casilla 170, Antofagasta, Chile

E-mail address: eortega@uantof.cl

Marko A. Rojas-Medar: Universidad Estadual de Campinas, Departamento de Matemática Aplicada, C.P. 6065, 13081-970, Campinas-SP, BraZIL

E-mail address: marko@ime.unicamp.br 


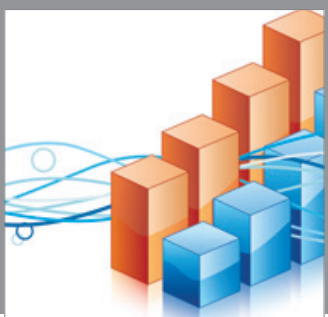

Advances in

Operations Research

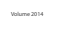

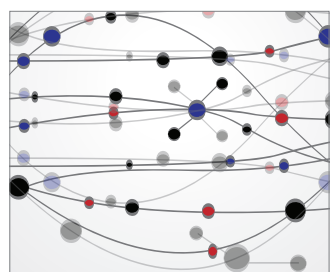

\section{The Scientific} World Journal
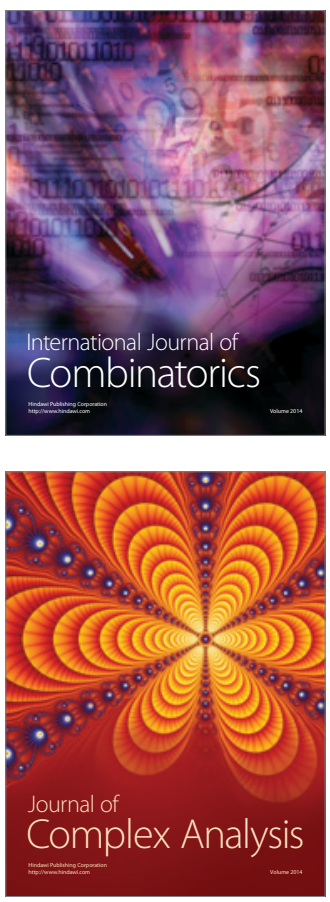

International Journal of

Mathematics and

Mathematical

Sciences
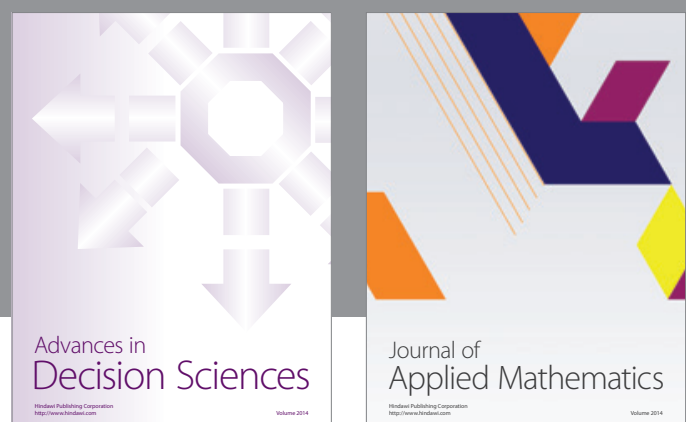

Journal of

Applied Mathematics
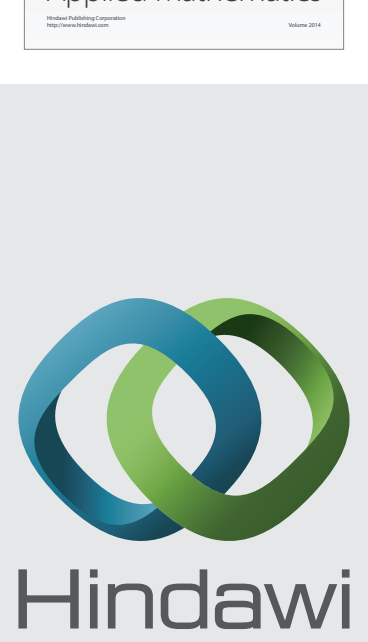

Submit your manuscripts at http://www.hindawi.com
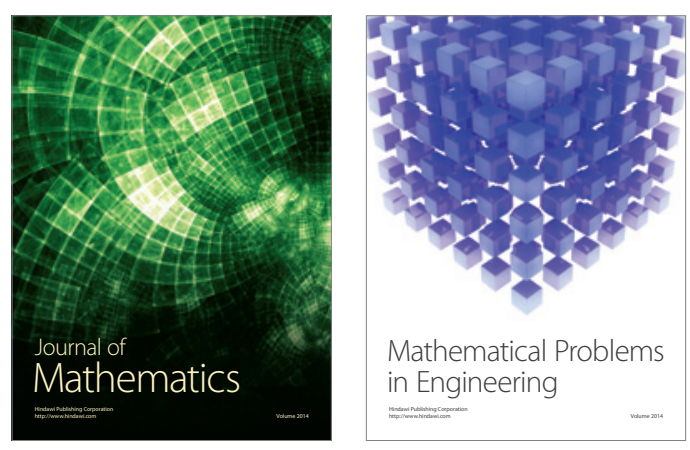

Mathematical Problems in Engineering
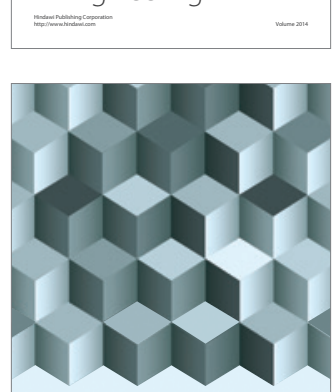

Journal of

Function Spaces
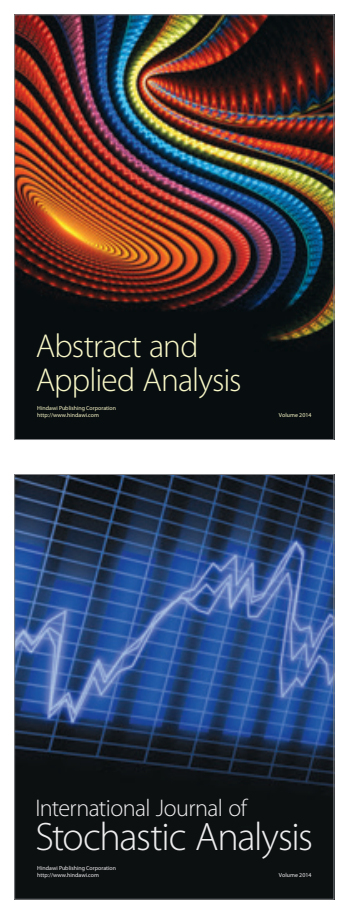

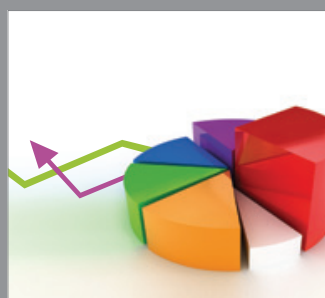

ournal of

Probability and Statistics

Promensencen
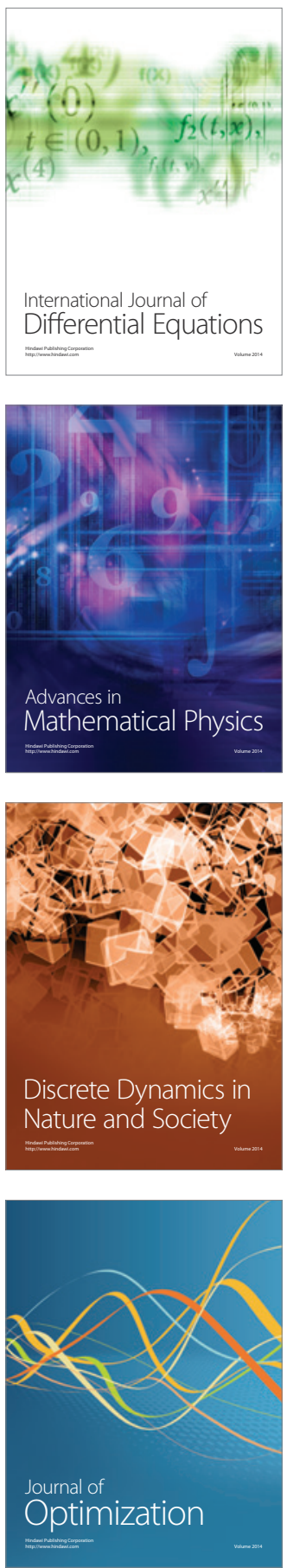